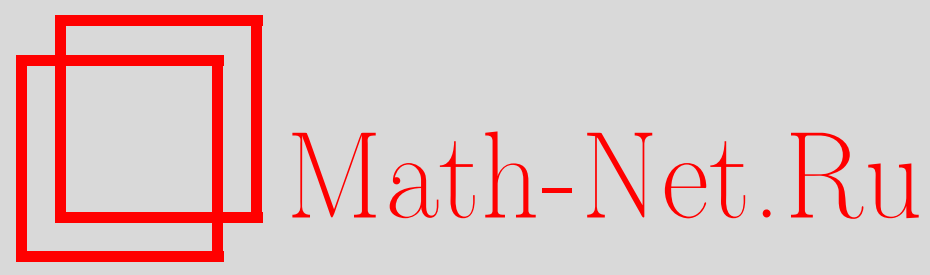

М. Г. Гимадисламов, О спектре дифференциального оператора высокого порядка, Матем. заметки, 2005, том 77, выпуск 2, 188-193

DOI: https://doi.org/10.4213/mzm2484

Использование Общероссийского математического портала Math-Net.Ru подразумевает, что вы прочитали и согласны с пользовательским соглашением http://www.mathnet.ru/rus/agreement

Параметры загрузки:

IP : 54.157 .27 .8

26 апреля 2023 г., 13:07:53

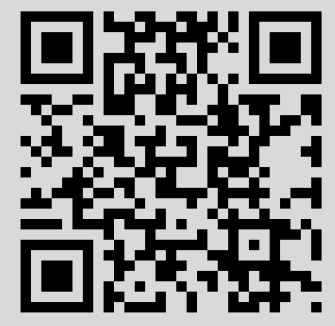




\section{О СПЕКТРЕ ДИФФЕРЕНЦИАЛЬНОГО ОПЕРАТОРА ВЫСОКОГО ПОРЯДКА}

\section{М.Г. Гимадисламов}

В статье для оператора высокого порядка получены достаточные условия дискретности и неограниченности снизу спектра этого оператора.

Библиограффия: 3 названия.

1. Рассматривается оператор $L$, заданный формулами

$$
\begin{gathered}
L y=(-1)^{r} y^{2 r}+q y, \quad 0 \leqslant x<\infty, \\
y^{(k)}(0)=0, \quad k=0,1, \ldots, r-1 .
\end{gathered}
$$

(Точнее, $L$ - это некоторое самосопряженное расширение оператора, заданное этими формулами на классе функций, обращающихся в нуль для достаточно больших $x$.) Здесь функция $q(x), x \in[0, \infty)$, предполагается вещественной и кусочно-непрерывной. Во многих работах выяснялись условия дискретности спектра оператора $L$. Обычно рассматривают случай $r=1$ и изучают оператор $L$ в одном из следуюших случаев: либо функция $q(x)$ ограничена снизу, либо быстро стремится к $-\infty$ (см. [1, с. 140], [2, с. 124]).

В работе Исмагилова [3] для случая $r=1$ был указан класс функций $q(x)$, не ограниченных ни сверху, ни снизу, для которых спектр оператора $L$ дискретен и не ограничен снизу. Доказательство, приведенное в [3], существенно использует специфику случая $r=1$; ниже мы объясним, что препятствует непосредственно обобщить это доказательство на случай $r>1$. Тем не менее, как будет показано, можно изменить рассуждения из [3] таким образом, что для $r>1$ получится результат, вполне аналогичный результатам для случая $r=1$. Опишем теперь основной результат.

Возьмем на $[0, \infty)$ точки $\left\{a_{k}\right\}, k \geqslant 1$, удовлетворяющие условиям

$$
\begin{gathered}
a_{1}<a_{2}<\cdots<a_{k}<\cdots, \quad a_{k} \rightarrow+\infty, \\
a_{k}-a_{k-1} \rightarrow 0 \quad \text { при } k \rightarrow \infty .
\end{gathered}
$$

Возьмем далее интервал $\Delta_{k}=\left(a_{k}-h_{k}, a_{k}+h_{k}\right)$, где числа $h_{k}$ положительны и столь малы, что эти интервалы попарно не пересекаются.

Рассмотрим кусочно-непрерывные вещественные функции $q(x), x \geqslant 0$, со следуюшими свойствами:

1) $q(x) \leqslant 0$ при $x \in \bigcup_{k=1}^{\infty} \Delta_{k}, q(x) \geqslant 0$ при $x \notin \bigcup_{k=1}^{\infty} \Delta_{k}$;

2) $h_{k}^{2 r-1} \int_{\Delta_{k}}|q(x)| d x \rightarrow+\infty$;

3) $\int_{\Delta_{k}}\left|x-a_{k}\right||q(x)| d x<\frac{1}{3}$. 
ТЕорема. Если функиия $q(x), x \in[0, \infty)$, удовлетворяет условиям 1)-3), то спектр оператора $L$ дискретен и неограничен снизу.

Подчеркнем, что жесткие условия на функцию $q(x)$ накладываются только на интервалах $\Delta_{k}$; вне этих интервалов $q(x)$ предполагается лишь неотрицательной (нет никаких дополнительных ограничений). Условия 2), 3) означают, грубо говоря, что функция $q(x), x \in \Delta_{k}$, сосредоточена в основном около точки $a_{k}$. Доказательство будет изложено в пІІ. 2 и 3.

2. Выясним свойства величин $\lambda_{n}^{L}(a, b)$.

Для любого отрезка $\Delta=[a, b] \subset[0, \infty)$ рассмотрим оператор $L$ в пространстве $L_{2}(a, b)$ с граничньми условиями Дирихле

$$
y^{(k)}(a)=y^{(k)}(b)=0, \quad k=0,1, \ldots, r-1 .
$$

Через $\lambda_{n}^{L}(\Delta)=\lambda_{n}^{L}(a, b)$ обозначим $n$-е собственное значение этого оператора, $n=$ $1,2, \ldots$ Величину $\lambda_{1}^{L}(\Delta)=\lambda_{1}^{L}(a, b)$ обозначим через $\lambda^{L}(\Delta)=\lambda^{L}(a, b)$. Из вариационного принципа Куранта вытекают следующие утверждения:

А) если для некоторого $n$-мерного подпространства $V_{n} \subset C_{0}^{\infty}(a, b)$ выполнено неравенство

$$
(L y, y) \leqslant C(y, y), \quad y \in V_{n},
$$

то $\lambda_{n}^{L}(a, b)<C$

B) $\lambda^{L}(a, b)=\inf \{(L y, y) /(y, y)\}, y \in C_{0}^{\infty}(a, b), y \neq 0$.

Зафиксируем на $[0, \infty)$ точки $a_{0}<a_{1}<\cdots<a_{n}$.

В следующей лемме содержатся неравенства, связьваюшие величины $\lambda_{n}^{L}\left(a_{k-1}, a_{k}\right)$.

ЛЕмма 1. 1) $E c л и \lambda^{L}\left(a_{k-1}, a_{k}\right) \leqslant C$ для любого $k=1, \ldots, n, m o$

$$
\lambda_{n}^{L}\left(a_{0}, a_{n}\right) \leqslant C .
$$

2) $\operatorname{Ecли~} \lambda^{L}\left(a_{k-1}, a_{k}\right) \geqslant d$ для любого $k=1, \ldots, n, m o$

$$
\lambda_{m}^{L}\left(a_{0}, a_{n}\right)>d,
$$

где $m=(n-1) r+1$.

ДокАЗАТЕЛЬСТво. 1) Пусть $\lambda^{L}\left(a_{k-1}, a_{k}\right) \leqslant C$ для любого $k=1, \ldots, n$. Тогда для любого $\varepsilon>0$ существуют такие $u_{k} \in C_{0}^{\infty}\left(a_{k-1}, a_{k}\right)$, что $\left\|u_{k}\right\|=1,\left(L u_{k}, u_{k}\right) \leqslant C+\varepsilon$, $k=1, \ldots, n$ (это следует из свойства В), приведенного вьше).

Пусть $V_{n}$-линейная оболочка системы $\left\{u_{k}, 1 \leqslant k \leqslant n\right\}$. Тогда ясно, что

$$
(L u, u) \leqslant(C+\varepsilon)(u, u) \quad \text { при } u \in V_{n} .
$$

Используя указанное вьше свойство А), видим, что $\lambda_{n}^{L}\left(a_{0}, a_{n}\right) \leqslant C+\varepsilon$. Так как $\varepsilon$ произвольно, то $\lambda_{n}^{L}\left(a_{0}, a_{n}\right) \leqslant C$.

2) Пусть $\lambda^{L}\left(a_{k-1}, a_{k}\right) \geqslant d$ для любого $k=1, \ldots, n$. Докажем, что

$$
\lambda_{m}^{L}\left(a_{0}, a_{m}\right) \geqslant d, \quad \text { где } d=(n-1) r+1 .
$$


Допустим, что $\lambda_{m}^{L}\left(a_{0}, a_{m}\right)<d$. Обозначим через $W_{m}$ линейную оболочку системы первых $m$ собственных функций оператора $L$ на отрезке $\left[a_{0}, a_{n}\right]$ (с граничньми условиями Дирихле). Так как $\operatorname{dim} W_{m}=m=(n-1) r+1$, существует такая функция $f \in W_{m}$, что $\|f\|=1, f^{(i)}\left(a_{k}\right)=0$ (здесь мы использовали тот факт, что количество ограничений, наложенных на функцию $f$, меньше размерности пространства $W_{m}$, откуда вытекает существование ненулевой функции $f$, удовлетворяющей этим ограничениям).

Итак, на каждом отрезке $\left[a_{k-1}, a_{k}\right]$ функция $f$ удовлетворяет граничным условиям Дирихле. Поэтому, обозначив через $f_{k}$ ограничение этой функции на отрезке $\left[a_{k-1}, a_{k}\right]$, получаем

$$
\left(L f_{k}, f_{k}\right) \geqslant d\left(f_{k}, f_{k}\right)
$$

Сложив эти неравенства, имеем

$$
(L f, f) \geqslant d(f, f) \text {. }
$$

С другой стороны,

$$
(L f, f) \leqslant \lambda_{m}^{L}(f, f) \quad \text { при } f \in W_{m},
$$

ибо $W_{m}$ - это линейная оболочка первых $m$ собственных функций оператора $L$ на $\left[a_{0}, a_{n}\right]$. Отсюда $\lambda_{m} \geqslant d$. Лемма 1 доказана.

Заметим, что если $r=1$, то величина $m=(n-1) r+1$, указанная в лемме, совпадает с $n$. Если же $r>1$, то $m>n$; это обстоятельство не позволяет применить лемму 1 (точнее ее второе утверждение) к доказательству теоремы. Чтобы преодолеть эту трудность, мы используем вместо второго утверждения леммы 1 следующую ниже лемму, в которой используется оператор $L_{0} y=-y^{\prime \prime}+q y$.

В этой лемме возникает также величина

$$
C_{r}=r^{-1 /(1-r)}-r^{-r /(1-r)} .
$$

Лемма 2. Пусть $\lambda^{L_{0}}\left(a_{k-1}, a_{k}\right) \geqslant d n p u k=1, \ldots, n$. Тогда

$$
\lambda_{n}^{L}\left(a_{0}, a_{n}\right) \geqslant d-C_{r}
$$

ДокАЗАТЕЛЬСТво. Используя лемму 1 при $r=1$, видим, что $\lambda^{L_{0}}\left(a_{0}, a_{n}\right) \geqslant d$. Чтобы получить аналогичную оценку для $\lambda^{L}\left(a_{0}, a_{n}\right)$, докажем неравенство

$$
(L y, y) \geqslant\left(L_{0} y, y\right)-C_{r}(y, y), \quad y \in C_{0}^{\infty}(0, \infty)
$$

Оно сводится к неравенству

$$
\left\|y^{(r)}\right\|^{2} \geqslant\left\|y^{\prime}\right\|^{2}-C_{r}\|y\|^{2}
$$

Для доказательства последнего неравенства переходим к преобразованию Фурье:

$$
\left\|y^{(r)}\right\|^{2}-\left\|y^{\prime}\right\|^{2}=\left\|x^{r} \widehat{y}\right\|^{2}-\|x \widehat{y}\|^{2}=\int_{0}^{\infty}\left(x^{2 r}-x^{2}\right)|\widehat{y}|^{2} d x .
$$


Здесь $\widehat{y}$ - преобразование Фурье функции $y(x)$. Найдя минимум функции $x^{2 r}-x^{2}$, получаем, что $x^{2 r}-x^{2} \geqslant-C_{r}$. Отсюда

$$
\left\|y^{(r)}\right\|^{2}-\left\|y^{\prime}\right\|^{2} \geqslant-C_{r}\|y\|^{2} .
$$

Этим доказано неравенство (3) и тем самым также неравенство (2). Теперь, используя вариационньй принцип Куранта, видим, что

$$
\lambda_{n}^{L}\left(a_{0}, a_{n}\right) \geqslant \lambda_{n}^{L_{0}}\left(a_{0}, a_{n}\right)-C_{r} \geqslant d-C_{r} .
$$

Лемма 2 доказана.

Следующая лемма дает признак дискретности и неограниченности снизу спектра оператора $L$, выраженньй посредством величин $\lambda^{L}(\Delta), \lambda^{L_{0}}(\Delta)$, где $\Delta$ - отрезок из $[0, \infty)$. В этой лемме мы фиксируем две последовательности $\left\{a_{k}\right\},\left\{b_{k}\right\}, k=1,2, \ldots$, такие, что $a_{1}<a_{2}<\cdots ; b_{1}<b_{2}<\cdots ; a_{k} \rightarrow+\infty, b_{k} \rightarrow+\infty$ при $k \rightarrow \infty$ и $b_{k}<a_{k}<b_{k+1}$ для всех $k$ (последнее условие означает, что эти последовательности перемежаются).

Лемма 3. Пусть $\lambda^{L_{0}}\left(a_{k}, a_{k+1}\right) \rightarrow+\infty u \lambda^{L}\left(b_{k}, b_{k+1}\right) \rightarrow-\infty n p u k \rightarrow \infty$. Тогдa спектр оператора $L$ дискретен и неограничен снизу.

ДокАЗАТЕЛЬСтво. Возьмем любое число $M>0$. Тогда найдется такой номер $m$, что

$$
\lambda^{L_{0}}\left(a_{k}, a_{k+1}\right)>M, \quad \lambda^{L}\left(b_{k}, b_{k+1}\right)<-M \quad \text { при } k \geqslant m+1 .
$$

Возьмем произвольное целое число $N>m$ и рассмотрим оператор $L$ на отрезке $\left[b_{m}\right.$, $\left.b_{m+N}\right)$ с граничными условиями Дирихле. По условию леммы

$$
\lambda^{L_{0}}\left(a_{k}, a_{k+1}\right)>M \quad \text { при } m-1 \leqslant k \leqslant m+N .
$$

Отсюда следует, что

$$
\lambda^{L_{0}}\left(b_{m}, b_{m+N}\right)>M, \quad \lambda^{L_{0}}\left(a_{m+N}, b_{m+N}\right) \geqslant M
$$

(ибо при сужении отрезка $\lambda^{L_{0}}$ увеличивается).

Применяя к отрезку $\left[b_{m}, b_{m+N}\right]$ лемму 2 , получаем неравенство

$$
\lambda_{N}^{L}\left(b_{m}, b_{m+N}\right) \geqslant M-C_{r} .
$$

Далее из неравенства $\lambda^{L}\left(a_{k}, a_{k+1}\right)<-M$ при $k=m, \ldots, m+N$ и из леммы 1 (точнее, ее первого утверждения) вытекает, что

$$
\lambda_{N}^{L}\left(a_{m}, a_{m+N}\right) \leqslant-M .
$$

Но тогда для большего отрезка $\left[b_{m}, b_{m+N}\right]$ получаем

$$
\lambda_{N}^{L}\left(b_{m}, b_{m+N}\right) \leqslant-M .
$$

Из неравенств $(4),(5)$ видно, что на интервале $\left[-M, M-C_{r}\right]$ нет ни одного собственного значения оператора $L$, рассматриваемого на отрезке $\left[b_{m}, b_{m+1}\right]$. Так как $N$ произвольно, то для оператора $L$, рассматриваемого на $\left[b_{m}, \infty\right)$ (с условиями Дирихле в точке $\left.b_{m}\right)$, любое самосопряженное расширение имеет конечньй спектр в интервале $(-M, M)$. Согласно принципу расщепления (см. [1, с. 41]) исходньй оператор $L$, рассматриваемый на $[0, \infty)$, имеет в $[-M, M]$ конечньй спектр. Так как $M$ произвольно, весь спектр дискретен. Наконец, из того, что $\lambda^{L}\left(b_{k}, b_{k+1}\right) \rightarrow-\infty$ при $k \rightarrow \infty$ вытекает, что спектр неограничен снизу. Лемма 3 доказана.

Заметим, что лемма 3 дает признак дискретности и неотрицательности спектра, которьй не эффективен (ибо использует величины $\lambda^{L}, \lambda^{L_{0}}$ вместо функции $q(x)$ ). В следующем пункте мы охарактеризуем спектр оператора $L$ при помощи функции $q(x)$. 
3. Доказательство теоремы. Пусть вьполнены предположения теоремы. Выберем точки $b_{k}$ так, что

$$
a_{k-1}+h_{k-1}<b_{k}<a_{k}+h_{k}
$$

Получили перемежающиеся последовательности $\left\{a_{k}\right\},\left\{b_{k}\right\}, k=1,2, \ldots$ Покажем, что для них вьполняются предположения леммы 3. Во-первьх, как доказано в [3],

$$
\lambda^{L_{0}}\left(a_{k-1}, a_{k}\right) \geqslant \frac{\pi^{2}}{3\left(a_{k}-a_{k-1}\right)^{2}}, \quad k=1,2, \ldots
$$

Так как $a_{k-1}-a_{k} \rightarrow 0$ при $k \rightarrow \infty$, то

$$
\lambda^{L_{0}}\left(a_{k-1}, a_{k}\right) \rightarrow+\infty \quad \text { при } \quad k \rightarrow \infty .
$$

Далее, оценим сверху величину $\lambda^{L}\left(b_{k}, b_{k+1}\right)$. Зафиксируем такую функцию $\varphi(x) \in$ $C_{0}^{\infty}(-1,1)$, что $\varphi(x) \geqslant 0,\|\varphi\|=1, \varphi(0)=1$. Зададим на отрезке $\Delta_{k}=\left(a_{k}-h_{k}, a_{k}+h_{k}\right)$ функцию

$$
\varphi_{k}(x)=\frac{1}{\sqrt{h_{k}}} \varphi\left(\frac{x-a_{k}}{h_{k}}\right) .
$$

Тогда $\varphi(x) \in C_{0}^{\infty}\left(\Delta_{k}\right),\|\varphi\|=1$. Поэтому, вводя обозначение

$$
\left\|\varphi^{(r)}\right\|^{2}=\Phi, \quad \max _{|x| \leqslant 1}|\varphi(x)|=Q,
$$

получаем

$$
\begin{aligned}
\lambda^{L}\left(b_{k}, b_{k+1}\right) & \leqslant \lambda^{L}\left(\Delta_{k}\right) \leqslant\left(L \varphi_{k}, \varphi_{k}\right)=\int_{\Delta_{k}}\left[\left|\varphi^{(r)}(x)\right|^{2}+\frac{1}{h_{k}} q(x)\left|\varphi\left(\frac{x-a_{k}}{h_{k}}\right)\right|^{2}\right] d x \\
& =\frac{\Phi}{h_{k}^{2 r}}+\frac{1}{h_{k}} \int_{\Delta_{k}} q(x) d x+\frac{1}{h_{k}} \int_{\Delta_{k}} q(x)\left(\left|\varphi\left(\frac{x-a_{k}}{h_{k}}\right)\right|^{2}-1\right) d x .
\end{aligned}
$$

Оценим последний интеграл. Положим

$$
S=\max _{|x| \leqslant 1}\left|\varphi^{\prime}(x)\right|
$$

В силу условия $\varphi(0)=1$ имеем (по формуле Лангранжа)

$$
\begin{aligned}
\left|\varphi\left(\frac{x-a_{k}}{h_{k}}\right)\right|^{2}-1 & =\left|\varphi\left(\frac{x-a_{k}}{h_{k}}\right)\right|^{2}-|\varphi(0)|^{2}=2\left|\varphi^{\prime}(c) \varphi(c)\right|\left|\frac{x-a_{k}}{h_{k}}\right| \\
& \leqslant 2 S Q\left|\frac{x-a_{k}}{h_{k}}\right|=T \frac{\left|x-a_{k}\right|}{h_{k}}, \quad \text { где } T=2 S Q .
\end{aligned}
$$

Отсюда, используя условие 3) теоремы, получаем

$$
\int_{\Delta_{k}} q(x)\left(\left|\varphi\left(\frac{x-a_{k}}{h_{k}}\right)\right|^{2}-1\right) d x \leqslant T h_{k}^{-1} \int_{\Delta_{k}}|q(x)|\left|x-a_{k}\right| d x<\frac{T}{3} h_{k}^{-1} .
$$


Итак,

$\lambda^{L}\left(b_{k}, b_{k+1}\right) \leqslant \frac{\Phi}{h_{k}^{2 r}}+\frac{1}{h_{k}} \int_{\Delta_{k}} q(x) d x+\frac{T}{3 h_{k}}=h_{k}^{-2 r}\left(\Phi+h_{k}^{2 r-1} \int_{\Delta_{k}} q(x) d x+\frac{1}{3} T h_{k}^{2 r-1}\right)$.

По условию 2) теоремы

$$
h_{k}^{2 r-1} \int_{\Delta_{k}} q(x) d x \rightarrow-\infty \quad \text { при } k \rightarrow \infty
$$

Отсюда

$$
\lambda^{L}\left(b_{k}, b_{k+1}\right) \rightarrow-\infty \quad \text { при } k \rightarrow \infty .
$$

Из (6), (7) видим, что выполнены условия леммы 3 . Тем самым, спектр оператора $L$ дискретен и неограничен снизу.

\section{СПИСОК ЦИТИРОВАННОЙ ЛИТЕРАТУРЫ}

[1] Глазман И. М. Прямые методы качественного спектрального анализа сингулярных дифференциальных операторов. М.: Физматгиз, 1963.

[2] Титчмарш Э. Ч. Разложение по собственным функциям, связанное с дифференциальными уравнениями второго порядка. Ч. 1. М.: ИЛ, 1960.

[3] Исмагилов Р. А. О спектре уравнения Штурма-Лиувилля с колеблющимся потенциалом // Матем. заметки. 1985. Т. 37. №6. С. 869-879.

Башкирский государственный университет, г. Уфа

Поступило

20.02 .2002

Исправленный вариант

28.03.2003 